\title{
Research on the Figure Module Integration Technology in Communication Monitoring
}

\author{
Li Wencui $^{1, a}$, Li Xiong ${ }^{1}$, Zhang Chi ${ }^{2}$, Wu Lijie ${ }^{1}$, Shu Xinjian ${ }^{1}$, Tang Weixia ${ }^{3}$ \\ ${ }^{1}$ Information \& Telecommunication Co. of State Grid Henan Electric Power Company, Zhengzhou, \\ 450052, China \\ ${ }^{2}$ Informational Engineering Department of Zhengzhou University, Zhengzhou 450001 China \\ ${ }^{3}$ China Information Technology Designing Consulting Institute Co. Ltd., Zhengzhou 450000 China) \\ aemail:elf8650@163.com
}

Keywords: interactive graphics sharing; figure module integration; comprehensive network; integrated monitoring

\begin{abstract}
Figure module integration technology is the graphics and graphical corresponding data in the database as a whole to face it. The graph model library integrated modeling method was researched in this paper. And by constructing communication network of vertical multi-dimensional comprehensive monitoring model, it can greatly reduce the generation and maintenance of network topology relationship of workload, quickly find locate abnormal events in a communication network.
\end{abstract}

\section{Introduction}

Traditional communication network topology graph modeling work is generally done by manual, in the face of complex electric power communication network topology, characteristics of various communications equipment, increasing scale, more and more graphics types and based on the monitoring and analysis of the graphics application, makes the establishment of the graphic communication network, it becomes difficult to update and accurate inspection, lead to high cost of operations, the efficiency is low. At the same time, the traditional professional network management tools only focus on the network operation monitoring of a single manufacturer, and the integrated network management tools only focus on the overall network running status, lack of vertical panorama of multi-dimensional comprehensive monitoring methods, a large number of decentralized communication equipment alarm information to the normal schedule on duty work, make the real-time monitoring of response ability is weak. Therefore, interactive graphics sharing and modulus of figure, the modulus of figure design and automatic mapping consistency, comprehensive network has become necessary to solve the key technology such as visual surveillance [1].

\section{Theoretical Foundation}

Figure Module Integration Technology Research. Figure module integration technology is the graphics and graphical corresponding data in the database as a whole to face it. Graphics is a form of data information, graphic image and visual expression of data information. A database is a tool for data storage, processing and management. Any system set up, the essence of which is its data relations and the establishment of a data structure. Has practical significance to make graphics and data, must be in accordance with the specific relationship bound them together. For graphics operations can be linked data and graphics, graphic attributes associated with data in the database, the data is modified and define or modify graphics and definition. Confirmed the graphics data is determined [2].

Users only need graphics operation, without the need to face the data behind it. Graphics operation is completed, the developed system can do not need to be built in human and modify records in the database, and in the same time, data binding and graphics, and is very convenient [3]. 
So figure module integration system is now developing graphics system is one of the most advanced technology.

Figure modulus of the integration of the key questions one is facing the is not only the modeling method of power equipment. The essence of it is that each equipment primitive objects and data in the database information corresponding to each one, in the system to a equipment primitive definition, at the same time, needs no human can increase the number of one or more database records. In the second, when users in the use of the software, equipment properties and parameters of the primitive can don't need a human can change, and the data input can also do this, so that the graphics and data in the database to one to one correspondence, but also to ensure that the software compatibility. Changes in equipment primitives, primitive parameters and attributes can change yourself, do not need human this is called automatic binding database, reduce the possibility of error. To figure and changes in the structure of the database table can not affect the graphic system and the purpose of graphic data, through the analysis of the atlas integration platform is divided into three modules: the graphic platform, primitive custom and custom attributes. The relations between and among as shown in the figure 1 below.

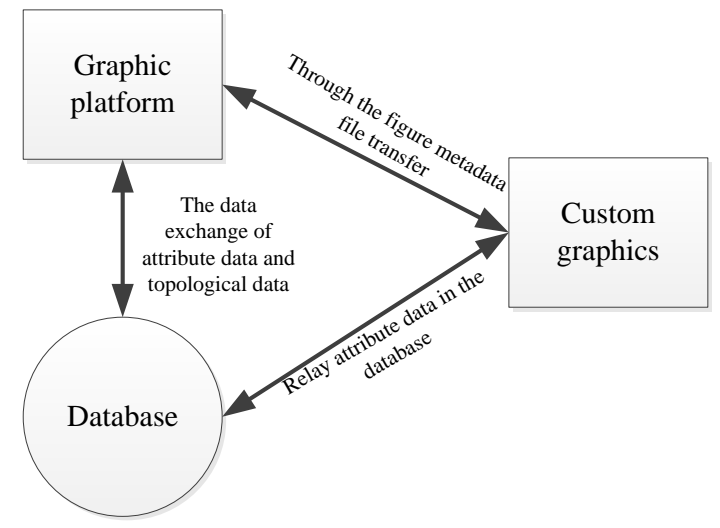

Fig.1 Figure module diagram

Big data technology refers to the huge amount of data from various types of fast access to valuable information technology. The core is big data technology to solve the problem of big data. Mainly can be divided into: data collection, data access, infrastructure, data processing and statistical analysis, data mining, model prediction, results of 8 kinds of technology. Big data technology mainly formed the batch, stream processing and interactive analysis of three kinds of calculation model [3].

1. Batch Processing technology represented by graphs and Hadoop system;

2. Stream Processing technology represented by Yahoo S4 system and Twitter Storm system;

3. Interactive Analysis technology represented by Google's Dremel system.

\section{The Communication Public Information Application Model}

The common information model is divided into the communication resources model, the repair order model, real-time data model [4]. The communication resource model is divided into physical resources model, logic model and business resources. Physical model from a physical point of view to describe the network resources and network resources, logical resource from the Angle of logic to describe all kinds of resources of network and network relations, business resource model based on the logical resource model, a unified description of all kinds of business resources and property model. See below public information model framework shown in Fig.2. 


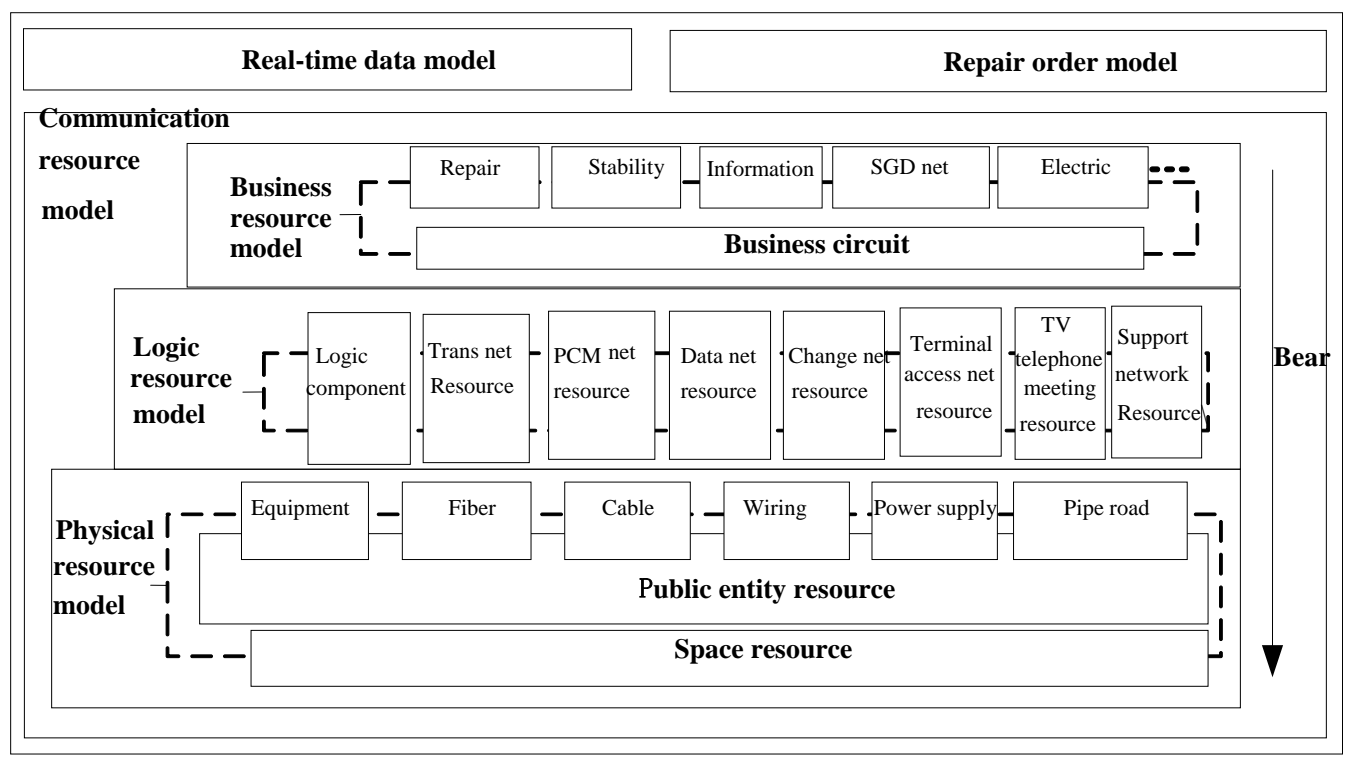

Fig.2 Common information model framework

On the classification of the common information model can be divided into spatial resources, equipment, components, wiring, communication power supply and line corridors resources, fiber optic cable, cable resources, logical components, transmission network, data network resources, PCM network resources, network resources, terminal communication access network resources, supporting network, emergency communications, television, telephone conference resources, etc.

On the common information model of correlation, in the management of communication resources, real-time monitoring, and operation of various objects relations has carried on the comprehensive analysis, the basic relation between communication object is given, and the relationship is divided into contain, bearing, link, inheritance, four parts:

Contain. An object is made up of other objects, such as: layout module contains multiple wiring terminals, transmission system, including multiple transmission equipment;

Bearing. Carrying an object, hold, placed on top of another object, such as cable section bearing in the pipe hole, circuit carries on the transmission channel;

Link. Two objects are connected together to form connections, such as cable section of the fiber core is connected to the ODF terminals, two time slot within a transmission equipment connected by cross configuration;

Inheritance. A child object derived from another parent object inheritance, all child objects inherited from the parent object characteristics and attributes, such as SDH transmission network inherited in the network.

The above research results as the figure modulus of the integration of research provides a solid theoretical and practical basis.

\section{Conclusion}

Traditional communication network topology graph modeling work is generally done by manual, in the face of complex electric power communication network topology, characteristics of various communications equipment, increasing scale, more and more graphics types and based on the monitoring and analysis of the graphics application, makes the establishment of the graphic communication network, it becomes difficult to update and accurate inspection, lead to high cost of operations, the efficiency is low.

\section{References}

[1] Xue Kui, He Shi-lin. The Research of Electric Information and Communication Risk Management[J]. Communication Technology, 2014,8. 
[2] Li Meng-xing, Chi Cheng-zhe, Wang Hai-yan. The Trend and Preventive Measures of Electric Information Safety Management[J], Power Information, 2010(8),12.

[3] Yang Bin, Zhang Da-hua, Xie Ying-jun. The Research and Design of Intelligent Electric Grid Communication Management System. Telecom Science, 2011,27(9A).

[4] Wang Ji-ye, Guo Jing-hong, Cao Jun-wei, Gao Ling-chao, Hu Zi-wei. Review on Information and Communication Key Technologies of Energy Internet[J].Smart Grid ,2015. 